\title{
Response to the letter to the editor by Tsatsos et al. "To remove or not to remove (the AC-IOL)? This is the question. Letter regarding article Endothelial keratoplasty in eyes with a retained angle-supported intraocular lens by Droutsas et al."
}

\author{
Konstantinos Droutsas • Apostolos Lazaridis - George Kymionis • \\ Klio Chatzistefanou - Dimitris Papaconstantinou - Walter Sekundo • \\ Chryssanthi Koutsandrea
}

Received: 19 August 2018/Accepted: 11 September 2018/Published online: 22 October 2018

(C) Springer Nature B.V. 2018

Dear Editor,

We thank colleagues Tsatsos et al. [1, 2] for sharing their experience on this controversial topic. Indeed, on the one hand, performing keratoplasty while leaving a stable anterior chamber intraocular lens (acIOL) in situ may be considered as the least invasive choice. On the other hand, exchanging an acIOL for a retroiridal one prior to keratoplasty restores the anterior segment anatomy to a better degree and may offer a longer graft survival.

The combination of endothelial keratoplasty with IOL exchange and fixation of the secondary IOL by gluing through scleral flaps as proposed by Narang et al. [2,3] is a promising new technique that has been performed on a small number of cases up to date. Thus, regardless of the fixation technique (i.e., sutured or glued), studies reporting long-term graft survival are

K. Droutsas $(\bowtie) \cdot$ G. Kymionis · K. Chatzistefanou •

D. Papaconstantinou · C. Koutsandrea

First Department of Ophthalmology, National and

Kapodistrian University of Athens, 11527 Athens, Greece

e-mail:kd2903@yahoo.gr

K. Droutsas · A. Lazaridis · W. Sekundo

Department of Ophthalmology, Philipps University,

35043 Marburg, Germany

G. Kymionis

Jules Gonin Eye Hospital, Faculty of Biology and

Medicine, University of Lausanne, Lausanne, Switzerland needed to confirm the superiority of sclera-fixated IOLs versus acIOLs.

We also agree with the comment of Tsatsos et al. regarding graft thickness in eyes with acIOL undergoing DSAEK. Indeed, as mentioned in "Results" section, we used thin or ultrathin DSAEK grafts with a diameter below $100 \mu \mathrm{m}$ [1].

Finally, also in our case series, a well-positioned acIOL and a deep anterior chamber were prerequisites for leaving an acIOL in situ as suggested by Esquenazi et al. [4].

\section{References}

1. Droutsas K, Lazaridis A, Kymionis G, Chatzistefanou K, Papaconstantinou D, Sekundo W, Koutsandrea C (2018) Endothelial keratoplasty in eyes with a retained angle-supported intraocular lens. Int Ophthalmol. https://doi.org/10. 1007/s10792-018-0899-x. (Epub ahead of print)

2. Tsatsos M, Athanasiadis I, Ziakas N (2018) To remove or not to remove (the AC-IOL)? This is the question. Int Ophthalmol. https://doi.org/10.1007/s10792-018-1021-0

3. Narang P, Agarwal A, Dua HS, Kumar DA, Jacob S, Agarwal A (2015) Glued intrascleral fixation of intraocular lens with pupilloplasty and pre-descemet endothelial keratoplasty: a triple procedure. Cornea 34(12):1627-1631

4. Esquenazi S, Esquenazi K (2010) Endothelial cell survival after descemet stripping with automated endothelial keratoplasty with retained anterior chamber intraocular lens. Cornea 29(12):1368-1372 